\title{
Illusion of Volume Loss
}

\author{
Neil A. Gordon, MD $^{1} \quad$ Julia Toman, MD $^{1}$ \\ ${ }^{1}$ Section of Otolaryngology, Head and Neck Surgery, Department of \\ Surgery, Yale University School of Medicine, Yale Physicians Building, \\ New Haven, Connecticut \\ Address for correspondence Neil A. Gordon, MD, Section of \\ Otolaryngology, Head and Neck Surgery, Department of Surgery, Yale \\ University School of Medicine, Yale Physicians Building, 4th Floor, 800 \\ Facial Plast Surg 2015;31:80-87. \\ Howard Avenue, New Haven, CT 06510 (e-mail: ng3364@aol.com).
}

\begin{abstract}
Keywords

- volume loss

- face-lift

- rhytidectomy

- aging face

- facial rejuvenation

Facial aging can create an appearance of volume loss and responds to volume enhancement in certain clinical scenarios. Actual fat loss is an illusion created by the inter-relationship of the different tissue types. The purpose of this article is to provide the anatomical, clinical, and research evidence to differentiate the contributions to facial aging from gravity's effects on soft tissue, fat loss, and skeletal remodeling, explaining the illusion.
\end{abstract}

It has been clearly stated in the literature for almost 50 years that changes in all four structural tissue types-skin, fat, muscle, and bone-contribute to facial aging. ${ }^{1}$ However, the extent to which changes in each of these tissues contribute to aging continue to be debated. The debate is complicated by the fact that proportional relationships can be deceiving as separate structures age and influence the perception of adjacent structures. Thus, an illusion can be created of a change in one tissue type where the change is actually in a different type (-Fig. 1).

As early as 1911 , volume restoration was advocated as a technique for facial rejuvenation. ${ }^{2,3}$ However, for most of the 20th century, the "surgical model" stated that the majority of facial aging was because of the gravity's effects on the facial soft tissue; therefore, lifting, repositioning, and excising excess soft tissue was the optimal solution to restore the face to its prior form. There were however, limitations and inadequacies in the esthetic outcomes, especially, when associated with techniques requiring skin tension, which lead to questioning of the "surgical model." The apparent inability of the "surgical model" to consistently address facial aging led to the recent popularity of the "volume model." This model emphasizes deflation and touts the resultant folds and hollows seen in the aging face as evidence of facial fat loss causing the majority of aging changes. Advances in soft tissue fillers and fat transfer techniques, as well as the ease, cost, and consumer demand for the "volume model" has fueled growth of this approach, despite similar evidence of limitations and inadequacies in esthetic outcomes.

Issue Theme Management of Facial Volume; Guest Editors, Edward D. Buckingham, MD, and Mark Glasgold, MD

Recently, several studies have documented the neglected contribution of the facial skeleton in understanding the aging process. ${ }^{4-7}$ By understanding the complex three-dimensional bony changes associated with the aging process, both the limitations of the surgical model and the successes of the volume model can be better understood.

It is our view, that facial aging is not because of significant facial fat loss, but is an illusion of loss produced by several factors. First, the normal physiology of soft tissue is to stretch secondary to intrinsic force, such as weight gain or pregnancy, and explains the success of tissue expansion techniques where extrinsic force is used to create soft tissue redundancy to repair soft tissue deficits. ${ }^{8}$ The extrinsic force of gravity on facial soft tissue also produces soft tissue redundancy, and differs in appearance from true cases of volume loss, such as HIV wasting syndrome, where the soft tissue retracts, resulting in an ill appearing rather than aged face. Second, the anatomical limitations restricting the ptotic, redundant facial soft tissue's movement produces similar hollows, folds, and signs of deflation in the face, mimicking volume depletion. Third, skeletal changes occur in the exact regions that volume enhancement have their greatest success: the medial cheek and periorbital region. In addition, the facial bone loss also explains the limitations in the "surgical model" approach to the aging face. We will present evidence to support these contributing factors that create the volume illusion and challenge the esthetic community to produce contradictory evidence of facial fat loss as the major contributor to the aged face.

Copyright @ 2015 by Thieme Medical Publishers, Inc., 333 Seventh Avenue, New York, NY 10001, USA. Tel: +1(212) 584-4662.
DOI http://dx.doi.org/ 10.1055/s-0035-1544250. ISSN 0736-6825. 


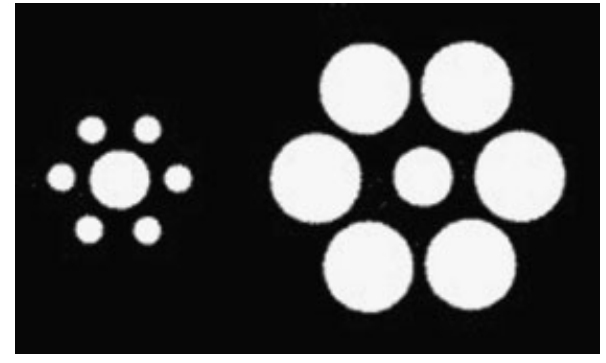

Fig. 1 Which center circle is larger? Both are the same. The illusion that the left center circle is larger is created by our brain's ability generalize relationships of adjacent structures from past experience.

\section{Relevant Anatomy}

The diagnosis and treatment of volume loss has mainly pertained to the periorbital and midface. For the purpose of our discussion, we will review midface anatomy. Viewing the

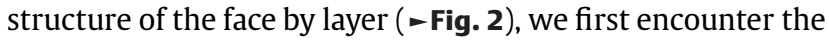
superficial soft tissue envelope which is bounded by the skin superficially and deeply by the submuscular aponeurotic system (SMAS) layer. This embryologic boundary is contiguous from the platysma in the neck to the galea in the forehead (-Fig.3). The fibrous septae which span the vertical thickness of this layer, are pathways for the vascular supply, coalesce into the facial retaining ligaments (zygomatic and mandibular), and compartmentalize what appears otherwise to be homogeneously distributed fat. ${ }^{9}$ The majority of the facial fat is distributed in the superficial soft tissue envelope. ${ }^{10}$

Below this layer is the superficial layer of the deep cervical fascia. The potential space located between these layers represents the embryologic cleavage plane of the midface and defines the deep plane. The three layers of the deep cervical fascia cover and envelope all the deeper structural elements of the face ( - Fig. 4) including the masseter muscle, facial nerve, major vasculature, and the deep facial fat pads. The anatomical description of the deep fat compartments vary according how the anatomy is defined. While Rohrich and Pessa ${ }^{9}$ describe the midface fat pads as nasolabial, medial, middle, and lateral cheek, as

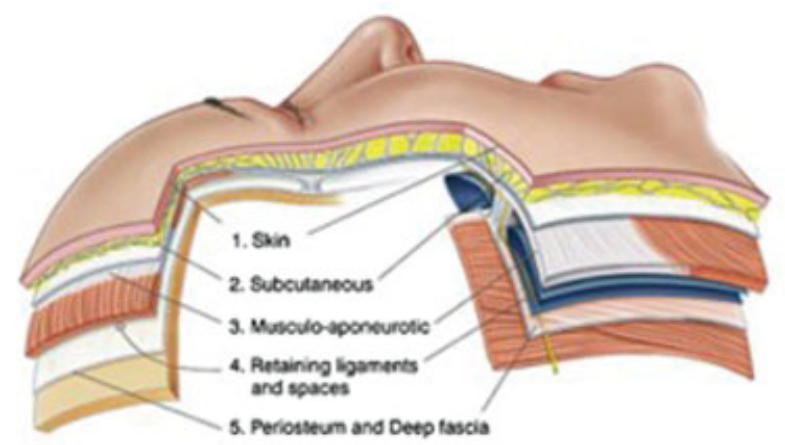

Fig. 2 Layers of the face. The superficial muscular aponeurotic system (SMAS) is the deep layer of the superficial soft tissue containing most of the facial fat distributed homogeneously superficial to the SMAS. (Adapted with permission from Mendelson BC, Jacobson SR. Surgical anatomy of the midcheek: facial layers, spaces, and midcheek segments. Clin Plast Surg 2008;35(3):398).

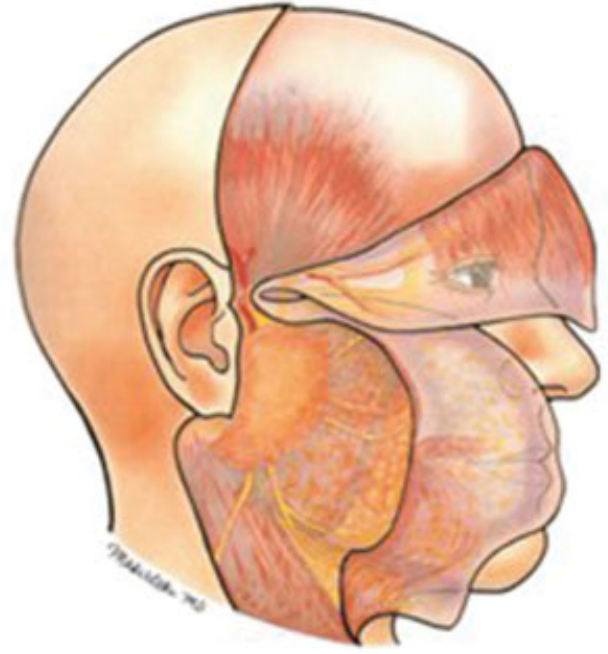

Fig. 3 The superficial soft tissue envelope bound superiorly by the skin and deeply by the superficial muscular aponeurotic system is an embryonic boundary contiguous from the platysma in the neck to the galea in the forehead. (Adapted with permission from Zoumalan RA, Larrabee WF. Anatomic considerations in the aging face. Facial Plast Surg 2011;27(1):18).

well as separate nasolabial and orbital compartments, the distinction between the compartments in the superficial soft tissue envelope versus the deep subfacial layer are not well defined. In Raskin and La Trenta's cadaver study, ${ }^{10}$ the layers of the face are better appreciated; the deep fat compartments are described as lacking uniformity with globules of cheek fat sparsely contained by fascia strands but also including defined structures such as the buccal fat and deep temporal pad.

These soft tissue layers rest on the periosteum encasing the facial skeleton.

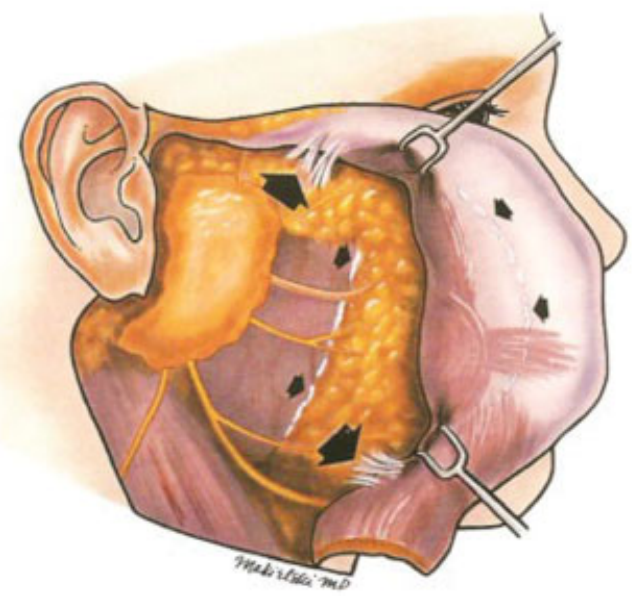

Fig. 4 Retaining ligaments of the face, with the superficial muscular aponeurotic system elevated. Both the zygomatic ligament (McGregor patch) superiorly, and the mandibular ligament inferiorly, are strong ligaments with periosteal attachments (wide arrows). Deep cervical fascia envelopes the underlying structural layers. (Adapted with permission from Zoumalan RA, Larrabee WF. Anatomic considerations in the aging face. Facial Plast Surg 2011;27(1):20). 


\section{Aging}

Clinical and research evidence show that the facial aging involves complex, multidimensional interactions between all the different tissue types. ${ }^{11}$ By understanding these relationships, we can better understand the degree of change in each tissue, and how changes in one tissue type can create the illusion of changes in a different tissue type. In addition, the success of certain clinical treatments, and failures or limitations of others, will also influence our current opinion of the predominant etiology of facial aging.

There is clear evidence that the soft tissues of our body were designed to respond to tensile force by stretching. ${ }^{8}$ The predominant factor in facial aging is caused by the slow insidious force of gravity and facial movement combining to create soft tissue redundancy in response to the stress. ${ }^{3,12}$ As the superficial soft tissue envelope of the face contains the majority of the facial fat and is poorly anchored to the underlying anatomy by two facial ligaments in the midface, the most significant effects of tensile force will be evident in this layer ${ }^{10}$ (-Fig. 4). However, as this layer is not free to descend in all the directions, as it is limited by the anatomical boundaries such as the nasolabial fold and the mandibular septum, as well as being influenced by other tissue layers, the soft tissue redundancy can "pile up" at these anatomical restriction areas creating folds and hollows mimicking volume depletion.

In HIV wasting syndrome, both lipodystrophy and lipoatrophy occur. Lipoatrophy specifically affects the midface creating medial hollows, prominent nasolabial folds, and skeletonized musculature. This creates an accurate appearance of the volume loss in the midface because of lipoatrophy. In true facial fat atrophy, hollows and folds are created without the evidence of soft tissue redundancy as would occur with aging. The look of HIV wasting syndrome is not of an aged individual, but of a sick, malnourished person (-Fig. $\mathbf{5}$ ).

To our knowledge, there has been no objective study defining facial fat loss because of aging, but cadaveric studies have been done that better define the anatomical relation-

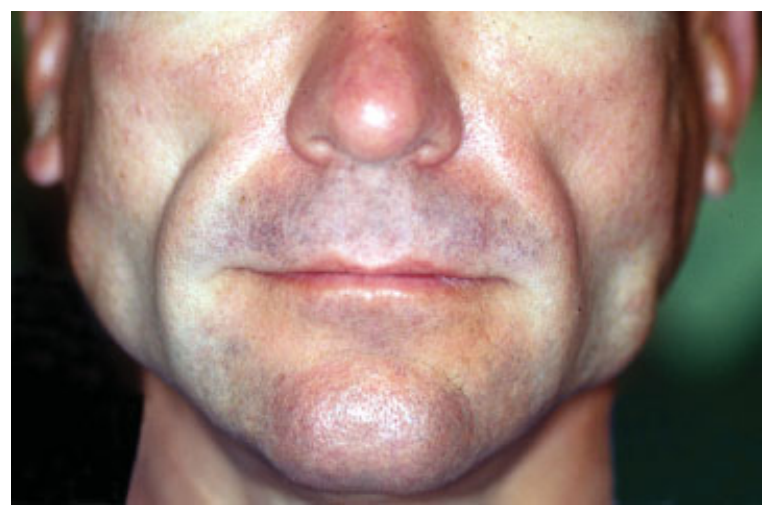

Fig. 5 Lipodystrophy in HIV wasting syndrome demonstrating true volume loss. (Image reprinted with permission from Medscape Reference [http://emedicine.medscape.com/] 2014. Available at: http:// emedicine.medscape.com/article/1082199-overview.) ships of facial fat compartments. These studies expose the difficulty in interpreting the relationships and influence that one tissue type has to an adjacent type.

Rohrich et al illustrated improvement of the "deflated midface" by the injection of $50 \mathrm{cc}$ of saline into the deep medial cheek pad and noted this as evidence of lipoatrophy as a cause of midface facial aging. ${ }^{13}$ As we will show, the tissue being replaced is not necessarily fat and the behavior of a cadaver's noncompliant soft tissue is not an accurate model to compare with the compliant soft tissue of the living.

In another cadaver study, Rohrich and Pessa demonstrated that although the superficial soft tissue envelope contains the majority of facial fat, it is not homogeneous but separated into units or compartments by fibrous septal bands. ${ }^{9}$ This, combined with the clinical observation of certain subunits, such as the medial cheek fat compartment preferentially appearing hollow, led to the conclusion that the fat compartments lose facial fat independent of one another.

If we view the superficial soft tissue envelope as a thick, quilted blanket, we can gain a better understanding of the illusion created by this study. If a blanket is lying on a flat, regular surface then the blanket will appear smooth or homogeneous. If something that is supporting the blanket is removed in only one area underneath, the contour of the blanket will change, but the true change in surface contour is not because of the blanket, but it is in the underlying support. We feel that the more logical answers as to why these illusions are created lies in understanding the changes in the bony facial skeleton that occur with aging.

In the last 15 years, a growing body of evidence has focused attention on facial skeletal remodeling and its effects on the aging face. Selective bone deposition and resorption is well documented in the facial skeleton and occurs regardless of the state of dentition, although loss of dentition significantly accelerates bone resorption of the maxilla and mandible. ${ }^{14,15}$ Using computed tomographic (CT) scans and other radiograph data, multiple authors have quantified the specific sites and relationship changes that occur because of bone resorption. ${ }^{4-7,16}$ By understanding these changes, the secondary effects on adjacent tissue types become evident.

In the periorbital region, the orbital aperture increases with age, mainly because of bone resorption in the superior/ medial and inferior/lateral orbit. Retrusion is also evident in the inferior/lateral region ${ }^{7}$ (-Figs. 6 and 7).

In the midface, evidence reveals that the maxilla both resorbs, causing the base of the pyriform aperture to increase in width, as well as retrudes with age causing loss of projection, regardless of dentition ${ }^{5}$ (-Figs. 6 and 8 ).

In the lower face, the height of the mandibular ramus and body decreases with age, especially with loss of dentition. In addition, retrusion occurs at the prejowl area $^{6}$ (- Figs. 6 and 9).

When viewing the facial skeleton from the lateral view, aging can be observed to cause remodeling with clockwise, inward rotation of the midface, decreasing projection in relation to the cranial skull base, confirming Lambros' theory of skeletal remodeling in the aging face ${ }^{17}$ (- Figs. 6 and 8 ). The consequences of the combined facial bone remodeling changes are significant and help explain both the limitations 


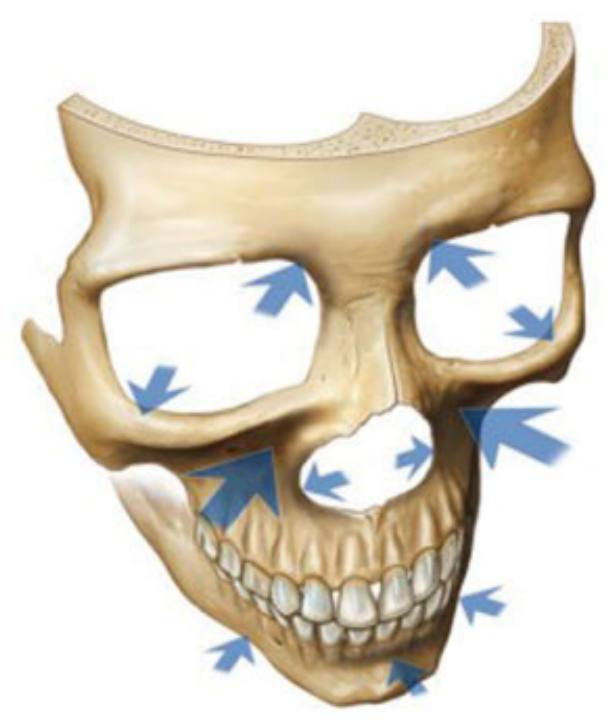

Fig. 6 Arrows indicate the areas of the facial skeleton susceptible to resorption with aging. The size of the arrow correlates with the amount of resorption. (Adapted with permission from Mendelson B, Wong C-H. Changes in the facial skeleton with aging: implications and clinical applications in facial rejuvenation. Aesth Plast Surg. 2012/08/01 2012;36(4):753-760).

of the "surgical model" and the success of the "volume model."

\section{Correlating These Bony Changes to Aging}

In the upper orbit, the superior medial bone loss explains the aging changes in the glabella as well as its resistance to treatment by brow lifting, especially in the elderly when skeletal changes are more advanced. In addition, this leads to a loss of fascial support and contributes to upper eyelid medial fat pseudoherniation. In the lower orbit, the inferior/lateral bone resorption and retrusion can cause pseudo fat herniation in the lower eyelid because of a posterior displacement of the orbital rim. It additionally explains the development of malar mounds or festoons, which are because of the loss of bony support for the orbicularis oculi muscle.
In the midface, generalized retrusion of the maxilla causes a loss of maxillary projection explaining the prominence of nasolabial folds and the tear trough deformity. In addition, the loss of fascial support causes buccal fat pseudoherniation, contributing to jowl formation. Combined with bone loss in the medial maxilla, the base of the pyriform aperture widens creating the appearance of the senile nose where a lack of support for the attachment of the lower lateral cartilages and nasal base leads to loss of projection and inferior rotation.

In the lower face, loss of mandibular height contributes to loss of support of the submandibular gland/digastric muscle triangle and exacerbates the ptotic, redundant neck softtissue by reducing the bony infrastructure. Retrusion at the prejowl region of the mandible also contributes to the appearance of jowls in the aging face.

\section{Discussion}

Lambros observed that the aged face when lifted in front of the mirror improved by layering out the excesses of soft tissue. ${ }^{3}$ This led to his generalization of all techniques of the "surgical model" in which soft tissue excesses were removed by traction. He questioned the validity of the "surgical model" because surgical outcomes did not approach the results of the "mirror lift." His dissatisfaction with the unnatural signs of "traction" on the skin and variability in cosmetic outcome led him to look at facial fat loss as the main etiology in facial aging, and emphasized volume enhancement as a priority in aging face treatment. ${ }^{3}$

A better understanding of the influence of procedure technique helps explain some of the perceived "surgical model" inadequacy. In SMAS rhytidectomy, the dissection is accomplished in the subcutaneous plane, disrupting the septal barriers between the fat compartments that directly connect the SMAS fascia to the skin, preventing the superficial soft tissue envelope from behaving as a unit. This disruption of normal anatomy limits soft tissue mobilization and repositioning, creating the necessity for skin traction as well as other inadequacies which lead Lambros to question the "surgical model."

In deep-plane rhytidectomy, the entire superficial soft tissue envelope is kept intact preserving the important

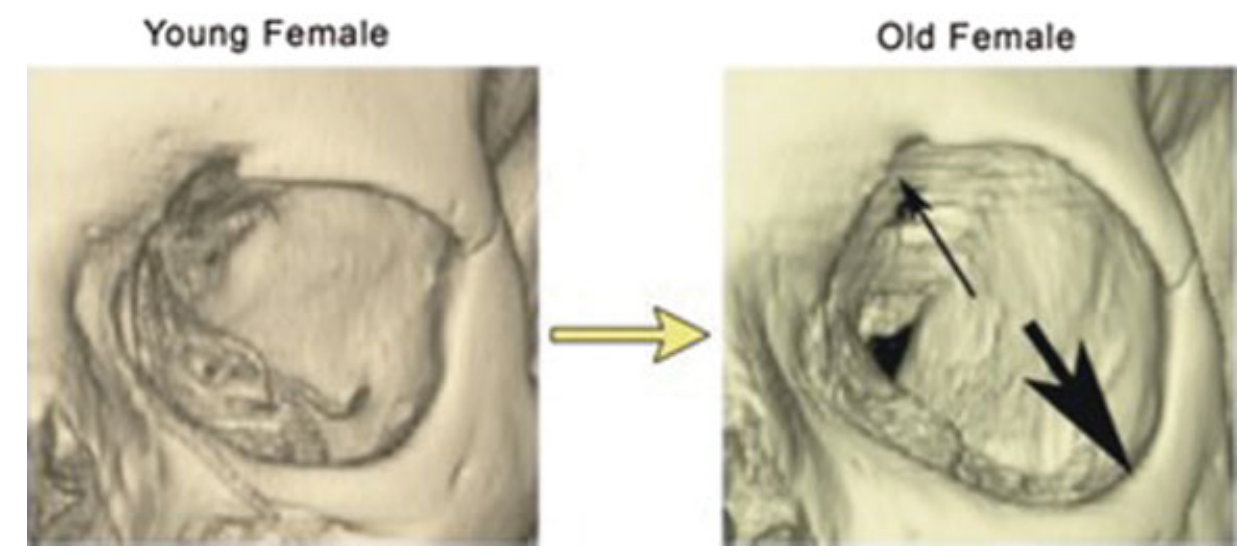

Fig. 7 Changes in bony orbit with age. (Adapted with permission from Shaw RB, Katzel EB, Koltz PF, Yaremchuk MJ, et al. Aging of the facial skeleton: aesthetic implications and rejuvenation strategies. Plast Reconstruct Surg. 2011;127(1):374-383). 

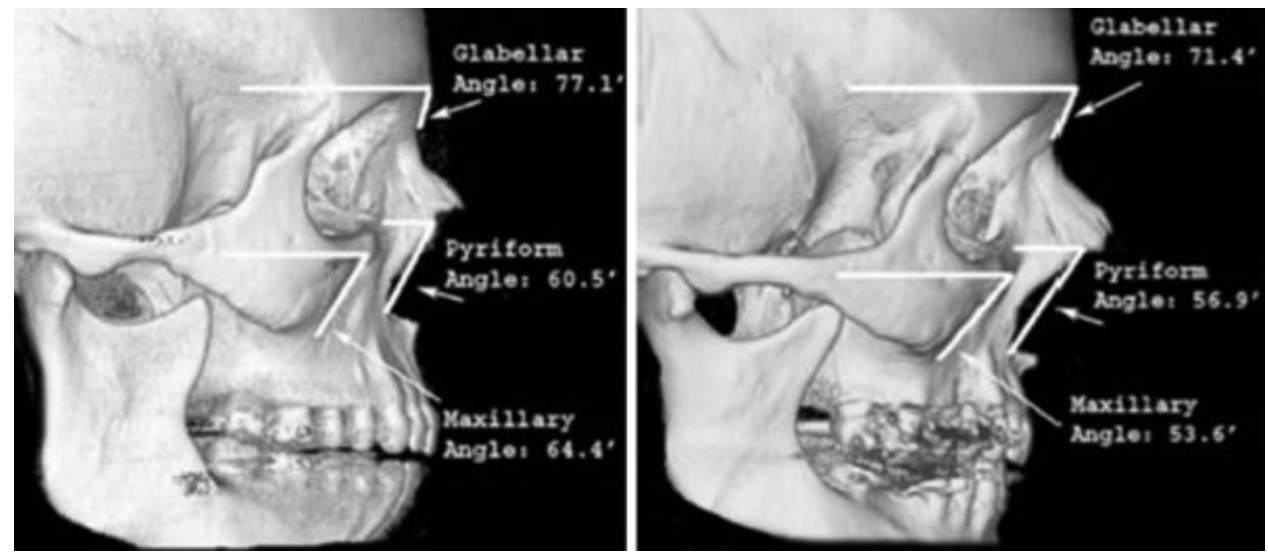

Fig. 8 The piriform (piriform angle) and the maxilla (maxillary angle) significantly recede with aging, from youth (left) to old age (right). (Adapted with permission from Shaw RBJ, Kahn DM. Aging of the midface bony elements: A three-dimensional computed tomographic study. Plast Reconstruct Surg. 2007;119(2):675-681).

structural anatomy. By keeping the fibrous septum connecting, the SMAS/platysma to the dermis intact, the superficial soft tissue envelope behaves as one unit ( - Fig. 10). Significant traction needs only to be applied to the deep SMAS/platysma layer, as it is connected to the dermis by these fibrous septae, causing the entire superficial soft tissue envelope to mobilize without any tension necessary at the skin level. This eliminates the unnatural surface changes and other inadequacies of the "surgical model" defined by Lambros. ${ }^{3}$ The entire superficial soft tissue envelope is mobilized by creating greater fat repositioning which "volumizes" the midface and maximizes the amount of soft tissue reduction necessary in reversing aging changes, creating outcomes that better duplicate the "mirror lift" (-Figs. 11 and 12).

Using intraoperative evidence from deep-plane rhytidectomies as the method to define the proportional contribution of gravity's effects to the facial aging process, we can see very significant amounts of soft tissue redundancy created both in obvious cases in the older patients, but also, surprisingly, in younger patients ( $\mathbf{- F i g . 1 3}$ ). The amount of soft tissue excess created by surgical facial degloving in the subfascial "deep" plane reveals gravity's significant effect in creating aging. The fact that younger patients also can exhibit large amounts of soft tissue redundancy points to soft-tissue compliance being a significant predictor of the rate of facial aging.

As soft-tissue redundancy cannot descend uninhibited because of the anatomical barriers such as the nasolabial fold, mandibular ligament, and the mandibular septum, the redundant soft tissue will "pile up" resulting in hollows and folds which mimic the appearance of facial fat loss. The illusion of fat loss is better understood by viewing in - Fig. 14. In the figure, there are two containers which each contain the same volume, the first figure is a smaller container (young face) and appears full. The same volume in a larger container (gravity-affected face) appears half empty or volume depleted. If we view the superficial soft tissue envelope as a container that holds the facial fat, the volume of fat can stay the same, but if the container increases in size because of the gravity's effects, an illusion of facial fat loss will occur.

Our recent understanding of facial skeletal changes provides the final piece of the puzzle. Loss of overall bony volume
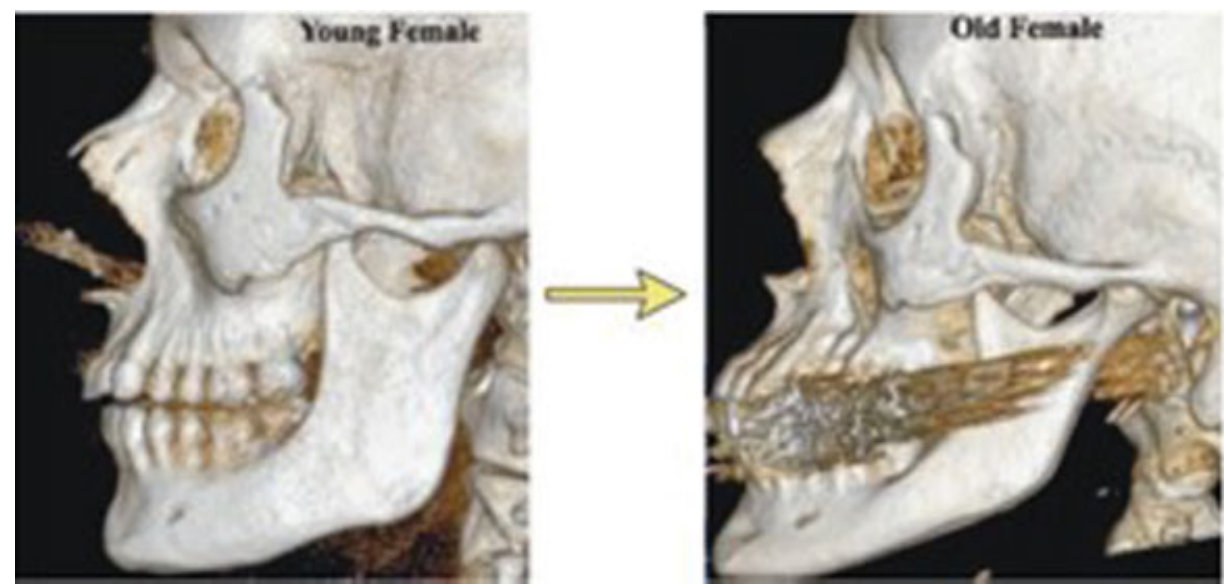

Fig. 9 Changes in bony mandible with age. (Adapted with permission from Shaw RB, Katzel EB, Koltz PF, Yaremchuk MJ, et al. Aging of the facial skeleton: aesthetic implications and rejuvenation strategies. Plast Reconstruct Surg. 2011;127(1):374-383). 


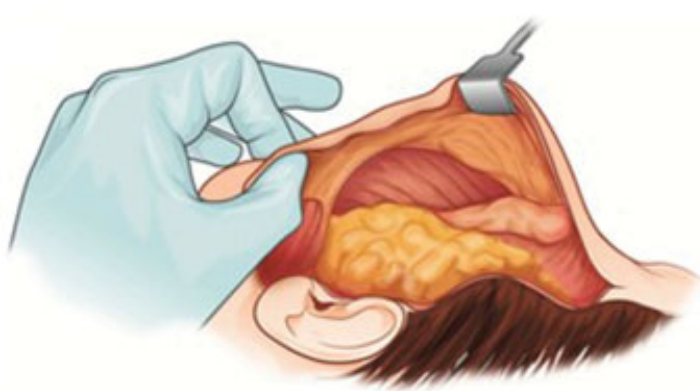

Fig. 10 A deep plan dissection schematically representing a fully degloved midface in the deep plane. The superficial soft tissue envelope contains the majority of the facial fat and behaves as a unit. (Adapted with permission from Gordon NA, Adam, SI. The deep plane approach to neck rejuvenation. Facial plastic surgery clinics of North America. 2014;22(2):269-284.)

enhances the overall appearance of soft tissue redundancy by shrinking the infrastructure over which the superficial soft tissue envelope rests, explaining the progression of neck changes in the advanced age. Specifically, the illusion of independent fat compartments aging in an otherwise homogeneous superficial soft tissue unit is better explained by understanding the changes in the structure that the soft tissue "blanket" rests on. Evidence from skeletal research shows one of the major areas of bone resorption and retrusion associated with aging is the medial maxilla. This is where clinical observation suggests the most obvious region of facial fat loss occurs. The bone resorption directly causes a loss of medial maxillary projection and increases the pyriform aperture both of which contribute to the illusion of independent fat compartment atrophy. The production of nasolabial folds and hollows, not fully reversible by the "surgical model," is more likely, in our opinion, proportional because of the skeletal loss rather than actual fat loss. In further support, the area also recalcitrant to the "surgical model," and simultaneously responsive to the "volume model," is the periorbital region. Here again, evidence points to significant skeletal resorption and loss of support structures as the predominant etiology.

While the proportion of aging changes because of the gravity's effects on the superficial soft-tissue envelope, which appear to be the predominant factor in aging, changes in the facial skeleton are significant and become more pronounced with advancing age. This helps explain the limitations in outcomes in certain older patients even with subfascial, soft-tissue reduction procedures.

A
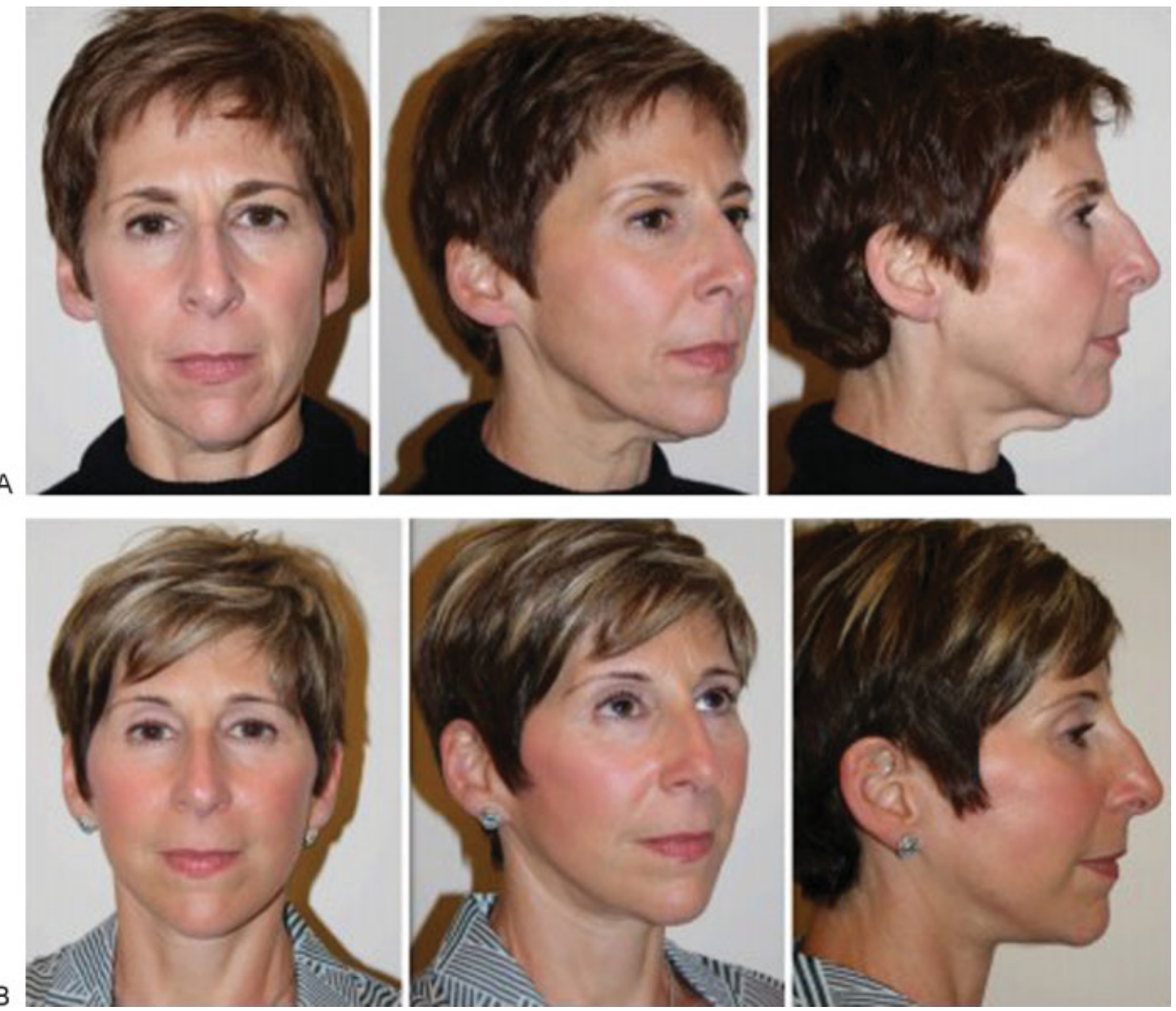

Fig. 11 Preoperative (A) and postoperative (B) photos of a 59-year-old woman who was treated with a deep plane facelift, browlift, upper and lower blepharoplasty, and periocular Er:YAG laser skin resurfacing. Note the recreation of her facial shape at both the midface and jawline with revolumization of the midface without the addition of extrinsic volume. 

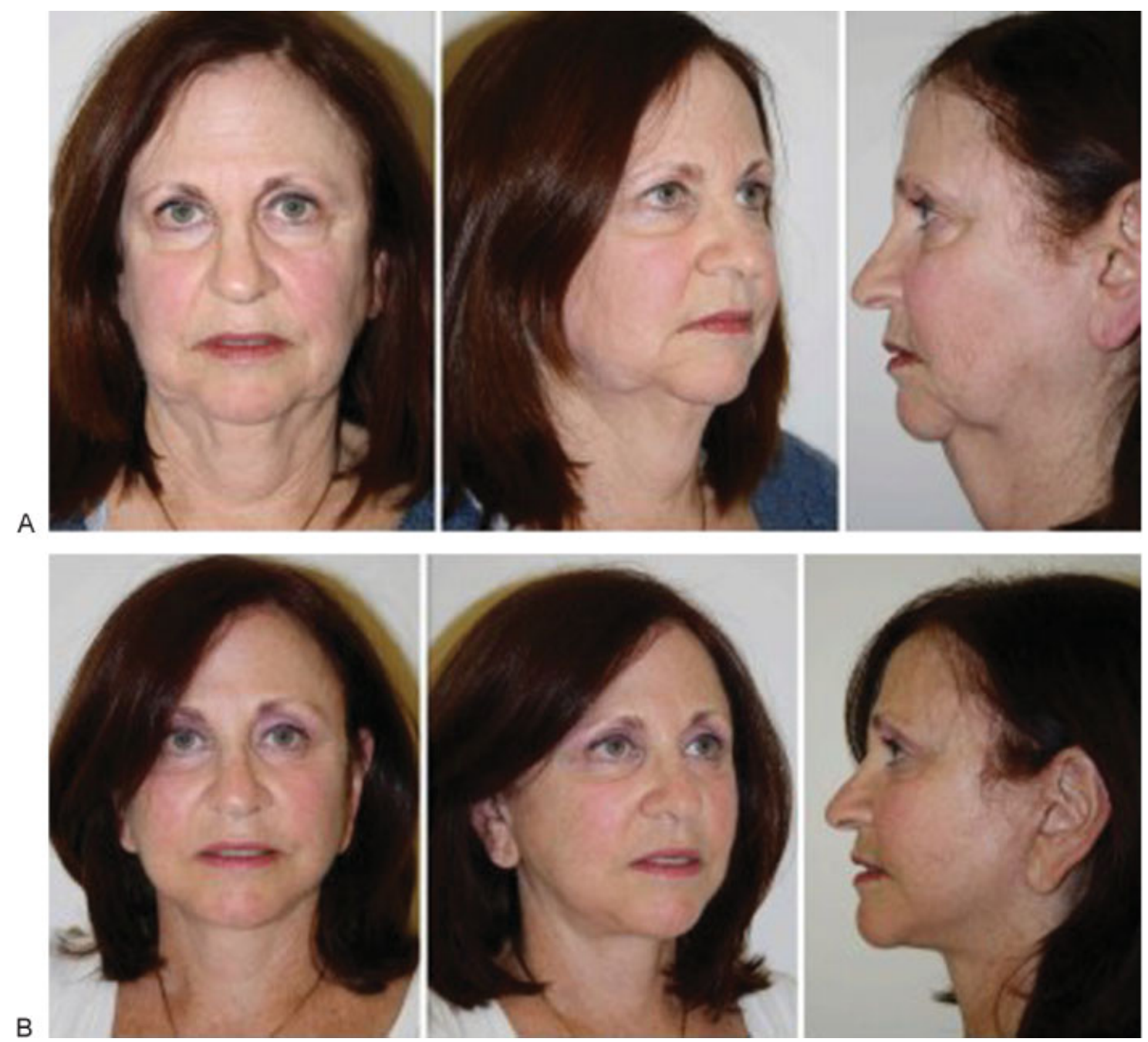

Fig. 12 Preoperative (A) and postoperative (B) photos of a 59-year-old woman who was treated with a deep-plane facelift, browlift, upper and lower blepharoplasty, and periocular and perioral Er:YAG laser skin resurfacing. Note the significance of gravity reversal, soft tissue reduction procedures in creating this outcome.

Volume enhancement is an essential part of facial rejuvenation. Its success as a treatment modality is best seen in younger patients where gravity changes are subtle and in areas of greatest facial bone resorption. The addition of volume is most likely camouflaging early gravity's effects on the soft tissues in younger patients, while restoring skeletal volume in advancing age. The use of volume enhancement is also successful in camouflaging certain inadequacies of soft tissue reduction procedures. Facial fat loss clearly can occur because of aging, but currently there is no study to our knowledge that documents these changes. As
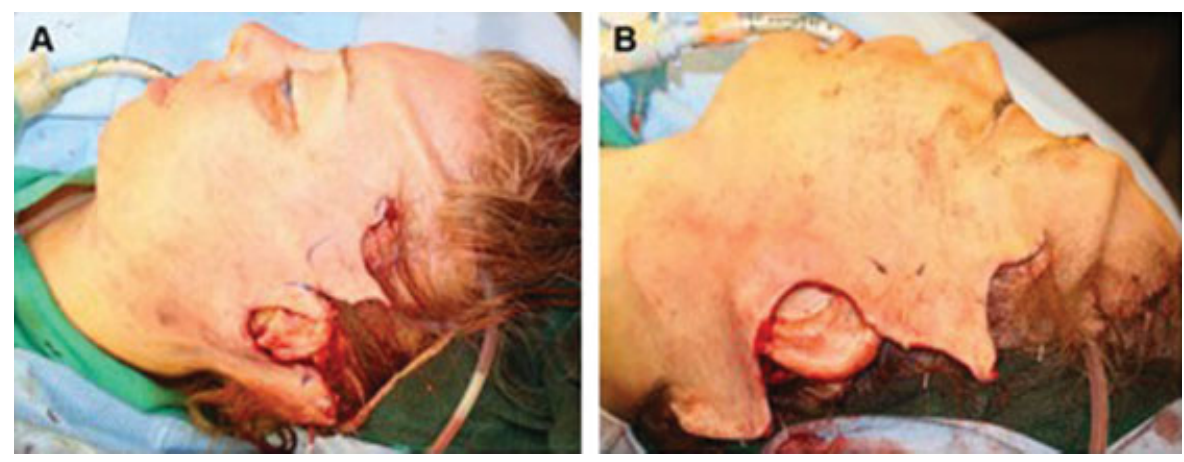

Fig. 13 Intraoperative photos demonstrating the excessive soft-tissue redundancy created after deep-plane dissection and mobilization of the facial soft tissues, even in the younger patient. This redundancy supports gravity's effects on facial soft tissue as the etiology in facial aging. (A) A 43-year-old woman undergoing deep plane facelift. (B) A 59-year-old woman undergoing deep plane facelift. 

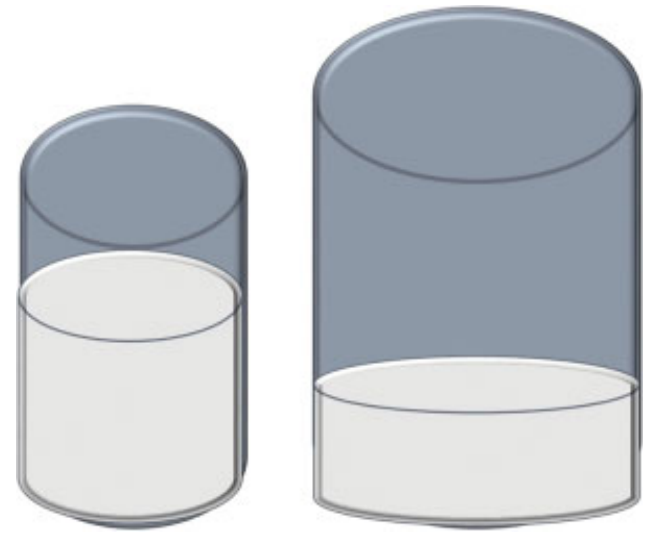

Fig. 14 Same volume but different size containers creates the illusion of greater volume in the smaller cylinder and less volume in the larger cylinder.

stated by Lambros, volume used as the sole treatment for the aging face just produces a "fat face."

\section{Conclusion}

Research and clinical evidence combine to reveal that gravity's effects on the superficial soft-tissue envelope of the face are the most significant effect in explaining the aged face. Skeletal remodeling and bone loss both enhance the appearance of gravity's effects, provide limitations to the surgical model of facial rejuvenation, and are likely being successfully treated by volume enhancement modalities. These effects combine to produce an illusion of facial fat loss as a major etiology in the aging face.

\section{Key Points}

1. Debate exists as to the etiology of facial aging with the "surgical model" emphasizing gravity's effects causing soft-tissue descent and ptosis while the "volume model" emphasizes deflation and fat loss.

2. The platysma muscle/SMAS/galea are the continuous superficial cervical fascia encompassing the majority of facial fat within the superficial soft tissue envelope, is poorly anchored to the face and most susceptible to gravity's stretching effects.

3. Research shows that the facial fat is compartmentalized but there is no direct evidence of facial fat loss because of aging.

4. Substantial evidence supports facial skeletal remodeling with bone loss in critical areas such as the orbit and maxilla explaining many aging changes.

5. Anatomical barriers impede the descent of soft tissue redundancy, creating hollows and folds, mimicking volume loss in the face.
6. Facial bone loss is greatest in regions most successfully treated with volume enhancement.

7. Facial aging is mainly because of gravity's long-term effects on the superficial soft tissue envelope, with increasing effects because of skeletal remodeling, especially with advanced age.

8. The visual effects of redundant soft tissue and selective bone loss create an illusion of facial fat loss being the significant contributor to the facial aging.

\section{References}

1 Gonzalez-Ulloa M, Flores ES. Senility of the face-basic study to understand its causes and effects. Plast Reconstr Surg 1965; 36:239-246

2 Kolle F. Plastic and cosmetic surgery. New York, NY: Appleton; 1911

3 Lambros V. Models of facial aging and implications for treatment. Clin Plast Surg 2008;35(3):319-327, discussion 317

4 Mendelson B, Wong C-H. Changes in the facial skeleton with aging: implications and clinical applications in facial rejuvenation. Aesthetic Plast Surg 2012;36(4):753-760

5 Shaw RBJ Jr, Kahn DM. Aging of the midface bony elements: a three-dimensional computed tomographic study. Plast Reconstr Surg 2007;119(2):675-681, discussion 682-683

6 Shaw RBJ Jr, Katzel EB, Koltz PF, Kahn DM, Girotto JA, Langstein HN. Aging of the mandible and its aesthetic implications. Plast Reconstr Surg 2010;125(1):332-342

7 Shaw RBJ Jr, Katzel EB, Koltz PF, et al. Aging of the facial skeleton: aesthetic implications and rejuvenation strategies. Plast Reconstr Surg 2011;127(1):374-383

8 Baker SR. Local Flaps in Facial Reconstruction. 3rd ed. Philadelphia, PA: Saunders; 2014

9 Rohrich RJ, Pessa JE. The retaining system of the face: histologic evaluation of the septal boundaries of the subcutaneous fat compartments. Plast Reconstr Surg 2008;121(5):1804-1809

10 Raskin E, Latrenta GS. Why do we age in our cheeks? Aesthet Surg J 2007;27(1):19-28

11 Fitzgerald R, Graivier MH, Kane M, et al. Update on facial aging. Aesthet Surg J 2010;30(Suppl):11S-24S

12 Gordon NA, Adam SI. The deep-plane approach to neck rejuvenation. Facial Plast Surg Clin North Am 2014;22(2):269-284

13 Rohrich RJ, Pessa JE, Ristow B. The youthful cheek and the deep medial fat compartment. Plast Reconstr Surg 2008;121(6): 2107-2112

14 Enlow DH. A morphogenetic analysis of facial growth. Am J Orthod 1966;52(4):283-299

15 Bolin A. Proximal alveolar bone loss in a longitudinal radiographic investigation. Swed Dent J Suppl 1986;35:1-108

16 Pessa JE, Desvigne LD, Lambros VS, Nimerick J, Sugunan B, Zadoo VP. Changes in ocular globe-to-orbital rim position with age: implications for aesthetic blepharoplasty of the lower eyelids. Aesthetic Plast Surg 1999;23(5):337-342

17 Pessa JE. An algorithm of facial aging: verification of Lambros's theory by three-dimensional stereolithography, with reference to the pathogenesis of midfacial aging, scleral show, and the lateral suborbital trough deformity. Plast Reconstr Surg 2000;106(2): 479-488, discussion 489-490 